\title{
A Note on Some Identities of New Type Degenerate Bell Polynomials
}

\author{
Taekyun Kim ${ }^{1,2, *}$, Dae San Kim ${ }^{3, *}$, Hyunseok Lee ${ }^{2}$ and Jongkyum Kwon ${ }^{4, *}$ \\ 1 School of Science, Xi'an Technological University, Xi'an 710021, China \\ 2 Department of Mathematics, Kwangwoon University, Seoul 01897, Korea; luciasconstant@gmail.com \\ 3 Department of Mathematics, Sogang University, Seoul 04107, Korea \\ 4 Department of Mathematics Education and ERI, Gyeongsang National University, \\ Gyeongsangnamdo 52828, Korea \\ * Correspondence: tkkim@kw.ac.kr (T.K.); dskim@sogang.ac.kr (D.S.K.); mathkjk26@gnu.ac.kr (J.K.)
}

Received: 24 October 2019; Accepted: 7 November 2019; Published: 11 November 2019

\begin{abstract}
Recently, the partially degenerate Bell polynomials and numbers, which are a degenerate version of Bell polynomials and numbers, were introduced. In this paper, we consider the new type degenerate Bell polynomials and numbers, and obtain several expressions and identities on those polynomials and numbers. In more detail, we obtain an expression involving the Stirling numbers of the second kind and the generalized falling factorial sequences, Dobinski type formulas, an expression connected with the Stirling numbers of the first and second kinds, and an expression involving the Stirling polynomials of the second kind.
\end{abstract}

Keywords: Bell polynomials; partially degenerate Bell polynomials; new type degenerate Bell polynomials

MSC: 05A19; 11B73; 11B83

\section{Introduction}

Studies on degenerate versions of some special polynomials can be traced back at least as early as the paper by Carlitz [1] on degenerate Bernoulli and degenerate Euler polynomials and numbers. In recent years, many mathematicians have drawn their attention in investigating various degenerate versions of quite a few special polynomials and numbers and discovered some interesting results on them [2-9]. This idea of introducing various degenerate versions of some special polynomials and numbers has been not only applied to polynomials but also extended to transcendental functions, so that for example, the degenerate gamma functions were introduced in $[10,11]$.

Indeed, for each real number $\lambda>0$, and any complex number $s$ satisfying $0<\operatorname{Re}(s)<\frac{1}{\lambda}$, the degenerate gamma function $\Gamma_{\lambda}(s)$ is defined by

$$
\Gamma_{\lambda}(s)=\int_{0}^{\infty}(1+\lambda t)^{-\frac{1}{\lambda} t^{s-1} d t .}
$$

Then, among many properties of them, let us mention only one of them, namely, for any positive integer $k$ and any real number $\lambda$ satisfying $0<\lambda<\frac{1}{k}$,

$$
\Gamma_{\lambda}(k)=\frac{(k-1) !}{(1-\lambda)(1-2 \lambda) \cdots(1-k \lambda)} .
$$

Bell polynomials $B_{n}(x)$ (see Equation (1)) are named after E. T. Bell [12] and also called exponential polynomials or Touchard polynomials. Bell polynomials together with their multivariate versions, 
namely the partial and complete Bell polynomials, have diverse applications not only in mathematics but also in physics and engineering as well [13].

For instance, the celebrated Faà di Bruno formula is given by

$$
\frac{d^{n}}{d t^{n}} f \circ g(t)=\sum_{k=0}^{n} f^{(k)}(g(t)) B_{n, k}\left(g^{\prime}(t), g^{\prime \prime}(t), \cdots, g^{(n-k+1)}(t)\right),
$$

which gives an explicit formula for higher derivatives of composite functions. Here the partial Bell polynomials $B_{n, k}\left(x_{1}, x_{2}, \cdots x_{n-k+1}\right)$ are defined by

$$
B_{n, k}\left(x_{1}, x_{2}, \cdots x_{n-k+1}\right)=\sum \frac{n !}{\prod_{l=1}^{n-k+1} i_{l} !} \prod_{l=1}^{n-k+1}\left(\frac{x_{l}}{l !}\right)^{i_{l}},(n \geq k \geq 0),
$$

where the sum runs over all nonnegative integers $i_{1}, i_{2}, \cdots, i_{n-k+1}$, satisfying $i_{1}+i_{2}+\cdots+i_{n-k+1}=k$, and $i_{1}+2 i_{2}+\cdots(n-k+1) i_{n-k+1}=n$, (see [13] p. 133).

In the previous paper, the partially degenerate Bell polynomials $\beta_{n, \lambda}(x)$, which are a degenerate version of Bell polynomials, were introduced (see Equation (3)) and some interesting identities on them were obtained in connection with Stirling numbers of the first and second kinds [14]. Further, in [15] the umbral calculus techniques were employed in order to derive some properties, explicit expressions and representations of them in terms of other special polynomials.

In the present paper, the new type degenerate Bell polynomials $B_{n}(x \mid \lambda)$, which are another degenerate version of Bell polynomials, are introduced and several expressions and identities on them are obtained.

As we have witnessed in recent years, studying some degenerate versions of certain special polynomials and numbers is a promising area of research that there are still many things yet to be uncovered.

In the rest of this section, we are going to review some necessary known definitions and results that will be needed throughout this paper.

As is well known, the ordinary Bell polynomials are given by (see $[12,14,16-21])$

$$
e^{x\left(e^{t}-1\right)}=\sum_{n=0}^{\infty} B_{n}(x) \frac{t^{n}}{n !}
$$

When $x=1, B_{n}=B_{n}(1)$ are called Bell numbers. For $\lambda \in \mathbb{R}$, the degenerate exponential function $e_{\lambda}^{x}(t)$ is defined as (see $\left.[1,2,4-7,10,11]\right)$

$$
e_{\lambda}^{x}(t)=(1+\lambda t)^{\frac{x}{\lambda}}, \quad e_{\lambda}(t)=e_{\lambda}^{1}(t)
$$

In [14], the partially degenerate Bell polynomials are defined by the generating function to be

$$
e^{x\left(e_{\lambda}(t)-1\right)}=\sum_{n=0}^{\infty} \beta_{n, \lambda}(x) \frac{t^{n}}{n !} .
$$

Note that $\lim _{\lambda \rightarrow 0} \beta_{n, \lambda}(x)=B_{n}(x),(n \geq 0)$. When $x=1, \beta_{n, \lambda}=\beta_{n, \lambda}(1)$ are called degenerate Bell numbers. It is well known that the Stirling polynomials of the second kind are defined by (see $[7,13])$

$$
(x+y)^{n}=\sum_{k=0}^{n} S_{2}(n, k \mid x)(y)_{n,} \quad(n \geq 0),
$$

where $(y)_{0}=1,(y)_{n}=y(y-1)(y-2) \cdots(y-n+1), \quad(n \geq 1)$. 
When $x=0, S_{2}(n, k)=S_{2}(n, k \mid 0),(n, k \geq 0)$, are called the Stirling numbers of the second kind. From Equation (4), we can easily derive the generating function for the Stirling polynomials of the second kind given by (see $[3,8,9,21,22])$

$$
\frac{1}{k !}\left(e^{t}-1\right)^{k} e^{x t}=\sum_{n=k}^{\infty} S_{2}(n, k \mid x) \frac{t^{n}}{n !},
$$

where $k$ is a nonnegative integer. Here we mention that the generating function has also played an important role in percolation theory. See the two seminal works $[23,24]$.

For $k \geq 0$, the central factorial numbers of the second kind are defined by (see $[2,5,13,21,25])$

$$
\frac{1}{k !}\left(e^{\frac{t}{2}}-e^{-\frac{t}{2}}\right)^{k}=\sum_{n=k}^{\infty} T(n, k) \frac{t^{n}}{n !} .
$$

In [7], Kim considered the degenerate Stirling polynomials of the second kind given by

$$
\frac{1}{k !}\left(e_{\lambda}(t)-1\right)^{k} e_{\lambda}^{x}(t)=\sum_{n=k}^{\infty} S_{2, \lambda}(n, k \mid x) \frac{t^{n}}{n !} .
$$

When $x=0, S_{2, \lambda}=S_{2, \lambda}(n, k \mid 0)$ are called the degenerate Stirling numbers of the second kind. In view of Equation (6), the degenerate central factorial numbers of the second kind are given by (see [5])

$$
\frac{1}{k !}\left(e_{\lambda}^{\frac{1}{2}}(t)-e_{\lambda}^{-\frac{1}{2}}(t)\right)^{k}=\sum_{n=k}^{\infty} T_{2, \lambda}(n, k) \frac{t^{n}}{n !} .
$$

Note that $\lim _{\lambda \rightarrow 0} T_{2, \lambda}(n, k)=T(n, k),(n, k \geq 0)$.

From Equations (1) and (5), we easily note that (see [21])

$$
B_{n}(x)=e^{-x} \sum_{m=0}^{\infty} x^{m} \frac{m^{n}}{m !}=\sum_{m=0}^{n} x^{m} S_{2}(n, m), \quad(n \geq 0) .
$$

For $n \geq 0$, the Stirling numbers of the first kind are defined as (see [21])

$$
(x)_{n}=\sum_{k=0}^{n} S_{1}(n, k) x^{k} .
$$

Thus, by Equation (10), we easily get (see [21])

$$
\frac{1}{k !}(\log (1+t))^{k}=\sum_{n=k}^{\infty} S_{1}(n, k) \frac{t^{n}}{n !},
$$

where $k$ is a nonnegative integer. It is easy to see that

$$
x^{n}=\sum_{k=0}^{n} S_{1}(n, k) B_{k}(x), \quad(k \geq 0) .
$$

From Equation (2), we can derive the inverse function of $e_{\lambda}(t)$ given by

$$
\log _{\lambda}(t)=\frac{1}{\lambda}\left(t^{\lambda}-1\right), \quad e_{\lambda}\left(\log _{\lambda}(t)\right)=t .
$$


By Equation (11), the degenerate Stirling numbers of the first kind are given by (see [4])

$$
\frac{1}{k !}\left(\log _{\lambda}(1+t)\right)^{k}=\sum_{n=k}^{\infty} S_{1, \lambda}(n, k) \frac{t^{n}}{n !},
$$

where $k$ is a nonnegative integer. Note that $\lim _{\lambda \rightarrow 0} S_{1, \lambda}(n, k)=S_{1}(n, k), \quad(n, k \geq 0)$.

In this paper, we study the new type degenerate Bell numbers and polynomials and we give some new identities for those polynomials and numbers.

\section{New Type Degenerate Bell Numbers and Polynomials}

For $\lambda \in \mathbb{R}$, the falling factorial sequence is defined by

$$
(x)_{0, \lambda}=1, \quad(x)_{n, \lambda}=x(x-\lambda) \cdots(x-(n-1) \lambda), \quad(n \geq 1) .
$$

From Equation (3), we note that

$$
e^{x t}=\sum_{m=0}^{\infty} \beta_{m, \lambda}(x) \frac{1}{m !}\left(\log _{\lambda}(1+t)\right)^{m}=\sum_{n=0}^{\infty}\left(\sum_{m=0}^{n} \beta_{m, \lambda}(x) S_{1, \lambda}(n, m)\right) \frac{t^{n}}{n !} .
$$

Thus, we have

$$
x^{n}=\sum_{m=0}^{n} \beta_{m, \lambda}(x) S_{1, \lambda}(n, m), \quad(n \geq 0) .
$$

On the other hand,

$$
e^{x\left(e_{\lambda}(t)-1\right)}=\sum_{m=0}^{\infty} x^{m} \frac{1}{m !}\left(e_{\lambda}(t)-1\right)^{m}=\sum_{n=0}^{\infty}\left(\sum_{m=0}^{n} x^{m} S_{2, \lambda}(n, m)\right) \frac{t^{n}}{n !} .
$$

By Equations (3) and (16), we get

$$
\beta_{n, \lambda}(x)=\sum_{m=0}^{n} x^{m} S_{2, \lambda}(n, m)=e^{-x} \sum_{m=0}^{\infty} x^{m} \frac{(m)_{n, \lambda}}{m !}, \quad(n \geq 0) .
$$

Indeed, from Equation (3), we have

$$
\begin{aligned}
\sum_{n=0}^{\infty} \beta_{n, \lambda}(x) \frac{t^{n}}{n !} & =e^{-x} e^{x e_{\lambda}(t)}=e^{-x} \sum_{m=0}^{\infty} \frac{1}{m !} x^{m} e_{\lambda}^{m}(t) \\
& =e^{-x} \sum_{m=0}^{\infty} \frac{1}{m !} x^{m}(1+\lambda t)^{\frac{m}{\lambda}} \\
& =e^{-x} \sum_{m=0}^{\infty} \frac{1}{m !} x^{m} \sum_{n=0}^{\infty}(m)_{n, \lambda} \frac{t^{n}}{n !} \\
& =\sum_{n=0}^{\infty}\left(e^{-x} \sum_{m=0}^{\infty} x^{m} \frac{(m)_{n, \lambda}}{m !}\right) \frac{t^{n}}{n !} .
\end{aligned}
$$


We observe that

$$
\begin{aligned}
\sum_{n=0}^{\infty} \beta_{n, \lambda}(x) \frac{t^{n}}{n !}=e^{x\left(e_{\lambda}(t)-1\right)} & =e^{x\left(e_{\lambda}^{1 / 2}(t)-e_{\lambda}^{-1 / 2}(t)\right) e_{\lambda}^{1 / 2}(t)} \\
& =\sum_{m=0}^{\infty} x^{m} \frac{1}{m !}\left(e_{\lambda}^{\frac{1}{2}}(t)-e_{\lambda}^{-\frac{1}{2}}(t)\right)^{m} e_{\lambda}^{\frac{m}{2}}(t) \\
& =\sum_{m=0}^{\infty} x^{m} \sum_{n=m}^{\infty}\left(\sum_{k=m}^{n}\left(\begin{array}{l}
n \\
k
\end{array}\right) T_{2, \lambda}(k, m)\left(\frac{m}{2}\right)_{n-k, \lambda}\right) \frac{t^{n}}{n !} \\
& =\sum_{n=0}^{\infty}\left(\sum_{m=0}^{n} \sum_{k=m}^{n}\left(\begin{array}{l}
n \\
k
\end{array}\right) x^{m} T_{2, \lambda}(k, m)\left(\frac{m}{2}\right)_{n-k, \lambda}\right) \frac{t^{n}}{n !} .
\end{aligned}
$$

Comparing the coefficients on both sides of Equation (18), we have

$$
\beta_{n, \lambda}(x)=\sum_{m=0}^{n} \sum_{k=m}^{n}\left(\begin{array}{l}
n \\
k
\end{array}\right) x^{m} T_{2, \lambda}(k, m)\left(\frac{m}{2}\right)_{n-k, \lambda}, \quad(n \geq 0) .
$$

Note that

$$
B_{n}(x)=\lim _{\lambda \rightarrow 0} \beta_{n, \lambda}(x)=\sum_{m=0}^{n} \sum_{k=m}^{n}\left(\begin{array}{l}
n \\
k
\end{array}\right) x^{m} T_{2}(k, m)\left(\frac{m}{2}\right)^{n-k} .
$$

Proposition 1. For $n \geq 0$, we have

$$
\beta_{n, \lambda}(x)=\sum_{m=0}^{n} \sum_{k=m}^{n}\left(\begin{array}{l}
n \\
k
\end{array}\right) x^{m} T_{2, \lambda}(k, m)\left(\frac{m}{2}\right)_{n-k, \lambda} .
$$

From (3), we have

$$
\begin{aligned}
e^{x\left(e^{t}-1\right)} & =\sum_{m=0}^{\infty} \beta_{m, \lambda}(x) \lambda^{-m} \frac{1}{m !}\left(e^{\lambda t}-1\right)^{m}=\sum_{m=0}^{\infty} \beta_{m, \lambda}(x) \lambda^{-m} \sum_{n=m}^{\infty} S_{2}(n, m) \lambda^{n} \frac{t^{n}}{n !} . \\
& =\sum_{n=0}^{\infty}\left(\sum_{m=0}^{n} \beta_{m, \lambda}(x) \lambda^{n-m} S_{2}(n, m)\right) \frac{t^{n}}{n !} .
\end{aligned}
$$

Therefore, by Equations (1) and (20), we obtain the following proposition.

Proposition 2. For $n \geq 0$, we have

$$
B_{n}(x)=\sum_{m=0}^{n} \beta_{m, \lambda}(x) \lambda^{n-m} S_{2}(n, m) .
$$

By Equation (1), we get

$$
\begin{aligned}
e^{x\left(e_{\lambda}(t)-1\right)} & =\sum_{m=0}^{\infty} B_{m}(x) \lambda^{-m} \frac{1}{m !}(\log (1+\lambda t))^{m} . \\
& =\sum_{m=0}^{\infty} B_{m}(x) \lambda^{-m} \sum_{n=m}^{\infty} S_{1}(n, m) \lambda^{n} \frac{t^{n}}{n !} \\
& =\sum_{n=0}^{\infty}\left(\sum_{m=0}^{n} B_{m}(x) \lambda^{n-m} S_{1}(n, m)\right) \frac{t^{n}}{n !} .
\end{aligned}
$$

Therefore, by Equations (3) and (21), we obtain the following proposition. 
Proposition 3. For $n \geq 0$, we have

$$
\beta_{n, \lambda}(x)=\sum_{m=0}^{n} B_{m}(x) \lambda^{n-m} S_{1}(n, m) .
$$

In view of Equation (3), we may define the new type degenerate Bell polynomials, $B_{n}(x \mid \lambda),(n \geq 0)$, by We would like to use the italic for the notation as a definition term.

$$
e_{\lambda}^{x}\left(e^{t}-1\right)=\left(1+\lambda\left(e^{t}-1\right)\right)^{\frac{x}{\lambda}}=\sum_{n=0}^{\infty} B_{n}(x \mid \lambda) \frac{t^{n}}{n !} .
$$

When $x=1, B_{n}(1 \mid \lambda)$ are called the new type degenerate Bell numbers. Note that

$$
\begin{aligned}
\sum_{n=0}^{\infty} \lim _{\lambda \rightarrow 0} B_{n}(x \mid \lambda) \frac{t^{n}}{n !} & =\lim _{\lambda \rightarrow 0} e_{\lambda}^{x}\left(e^{t}-1\right)=\lim _{\lambda \rightarrow 0}\left(1+\lambda\left(e^{t}-1\right)\right)^{\frac{x}{\lambda}} \\
& =e^{x\left(e^{t}-1\right)}=\sum_{n=0}^{\infty} B_{n}(x) \frac{t^{n}}{n !}
\end{aligned}
$$

By comparing the coefficients on both sides, we get

$$
\lim _{\lambda \rightarrow 0} B_{n}(x \mid \lambda)=B_{n}(x), \quad(n \geq 0) .
$$

By Equation (22), we get

$$
\begin{aligned}
e_{\lambda}^{x}\left(e^{t}-1\right) & =\left(1+\lambda\left(e^{t}-1\right)\right)^{\frac{x}{\lambda}}=\sum_{k=0}^{\infty}(x)_{k, \lambda} \frac{1}{k !}\left(e^{t}-1\right)^{k} \\
& =\sum_{k=0}^{\infty}(x)_{k, \lambda} \sum_{n=k}^{\infty} S_{2}(n, k) \frac{t^{n}}{n !} \\
& =\sum_{n=0}^{\infty}\left(\sum_{k=0}^{n}(x)_{k, \lambda} S_{2}(n, k)\right) \frac{t^{n}}{n !} .
\end{aligned}
$$

Therefore, by Equations (22) and (23), we obtain the following theorem.

Theorem 1. For $n \geq 0$, we have

$$
B_{n}(x \mid \lambda)=\sum_{k=0}^{n} S_{2}(n, k)(x)_{k, \lambda}
$$

In particular,

$$
B_{n}(1 \mid \lambda)=\sum_{k=0}^{n} S_{2}(n, k)(1)_{k, \lambda}
$$


We observe that

$$
\begin{aligned}
e_{\lambda}^{x}\left(e^{t}-1\right) & =e^{\frac{x}{\lambda} \log \left(1+\lambda\left(e^{t}-1\right)\right)}=\sum_{m=0}^{\infty}\left(\frac{x}{\lambda}\right)^{m} \frac{1}{m !}\left(\log \left(1+\lambda\left(e^{t}-1\right)\right)\right)^{m} \\
& =\sum_{m=0}^{\infty}\left(\frac{x}{\lambda}\right)^{m} \sum_{l=m}^{\infty} S_{1}(l, m) \lambda^{l} \frac{1}{l !}\left(e^{t}-1\right)^{l} \\
& =\sum_{l=0}^{\infty} \sum_{m=0}^{l} x^{m} \lambda^{l-m} S_{1}(l, m) \sum_{n=l}^{\infty} S_{2}(n, l) \frac{t^{n}}{n !} \\
& =\sum_{n=0}^{\infty}\left(\sum_{l=0}^{n} \sum_{m=0}^{l} x^{m} \lambda^{l-m} S_{1}(l, m) S_{2}(n, l)\right) \frac{t^{n}}{n !}
\end{aligned}
$$

Therefore, by Equations (22) and (24), we obtain the following theorem.

Theorem 2. For $n \geq 0$, we have

$$
B_{n}(x \mid \lambda)=\sum_{l=0}^{n} \sum_{m=0}^{l} x^{m} \lambda^{l-m} S_{1}(l, m) S_{2}(n, l) .
$$

In particular,

$$
B_{n}(1 \mid \lambda)=\sum_{l=0}^{n} \sum_{m=0}^{l} \lambda^{l-m} S_{1}(l, m) S_{2}(n, l)
$$

From Equation (22), we note that

$$
\begin{aligned}
\sum_{n=0}^{\infty} B_{n}(x \mid \lambda) \frac{t^{n}}{n !} & =e_{\lambda}^{x}\left(e^{t}-1\right)=\left(1+\lambda\left(e^{t}-1\right)\right)^{\frac{x}{\lambda}} \\
& =\sum_{l=0}^{\infty}(x)_{l, \lambda} \frac{1}{l !}\left(e^{t}-1\right)^{l}=\sum_{l=0}^{\infty}(x)_{l, \lambda} \frac{1}{l !} \sum_{m=0}^{l}\left(\begin{array}{c}
l \\
m
\end{array}\right)(-1)^{l-m} e^{m t} \\
& =\sum_{l=0}^{\infty}(x)_{l, \lambda} \frac{1}{l !} \sum_{m=0}^{l}\left(\begin{array}{c}
l \\
m
\end{array}\right)(-1)^{l-m} \sum_{n=0}^{\infty} m^{n} \frac{t^{n}}{n !} \\
& =\sum_{n=0}^{\infty}\left(\sum_{l=0}^{\infty} \sum_{m=0}^{l} \frac{1}{l !}\left(\begin{array}{c}
l \\
m
\end{array}\right)(-1)^{l-m}(x)_{l, \lambda} m^{n}\right) \frac{t^{n}}{n !}
\end{aligned}
$$

Comparing the coefficients on both sides of Equation (25), we obtain the following theorem.

Theorem 3 (Dobiniski type formula). For $n \geq 0$, we have

$$
B_{n}(x \mid \lambda)=\sum_{l=0}^{\infty} \sum_{m=0}^{l} \frac{1}{l !}\left(\begin{array}{c}
l \\
m
\end{array}\right)(-1)^{l-m}(x)_{l, \lambda} m^{n}
$$

In particular,

$$
B_{n}(1 \mid \lambda)=\sum_{l=0}^{\infty} \sum_{m=0}^{l} \frac{1}{l !}\left(\begin{array}{c}
l \\
m
\end{array}\right)(-1)^{l-m}(1)_{l, \lambda} m^{n}
$$

Note that

$$
\lim _{\lambda \rightarrow 0} B_{n}(x \mid \lambda)=\sum_{l=0}^{\infty} \sum_{m=0}^{l} \frac{1}{l !}\left(\begin{array}{c}
l \\
m
\end{array}\right)(-1)^{l-m} x^{l} m^{n}=e^{-x} \sum_{k=0}^{\infty} \frac{k^{n}}{k !} x^{k}=B_{n}(x) .
$$


By Equation (22), we get

$$
\begin{aligned}
\sum_{n=1}^{\infty} B_{n}(x \mid \lambda) \frac{t^{n-1}}{(n-1) !} & =\frac{\partial}{\partial t} e_{\lambda}^{x}\left(e^{t}-1\right)=\frac{\partial}{\partial t}\left(1+\lambda\left(e^{t}-1\right)\right)^{\frac{x}{\lambda}} \\
& =x e^{t}\left(1+\lambda\left(e^{t}-1\right)\right)^{\frac{x-\lambda}{\lambda}}=x e^{t} e_{\lambda}^{x-\lambda}\left(e^{t}-1\right) \\
& =x \sum_{l=0}^{\infty}(x-\lambda)_{l, \lambda} \frac{1}{l !}\left(e^{t}-1\right)^{l} \sum_{m=0}^{\infty} \frac{t^{m}}{m !} \\
& =x \sum_{l=0}^{\infty}(x-\lambda)_{l, \lambda} \sum_{k=l}^{\infty} S_{2}(k, l) \frac{t^{k}}{k !} \sum_{m=0}^{\infty} \frac{t^{m}}{m !} \\
& =x \sum_{k=0}^{\infty} \sum_{l=0}^{k}(x-\lambda)_{l, \lambda} S_{2}(k, l) \frac{t^{k}}{k !} \sum_{m=0}^{\infty} \frac{t^{m}}{m !} \\
& =\sum_{n=0}^{\infty}\left(x \sum_{k=0}^{n} \sum_{l=0}^{k}\left(\begin{array}{l}
n \\
k
\end{array}\right)(x-\lambda)_{l, \lambda} S_{2}(k, l)\right) \frac{t^{n}}{n !} .
\end{aligned}
$$

Thus, we have

$$
\sum_{n=0}^{\infty} B_{n+1}(x \mid \lambda) \frac{t^{n}}{n !}=\sum_{n=0}^{\infty}\left(x \sum_{k=0}^{n} \sum_{l=0}^{k}\left(\begin{array}{l}
n \\
k
\end{array}\right)(x-\lambda)_{l, \lambda} S_{2}(k, l)\right) \frac{t^{n}}{n !} .
$$

Therefore, by comparing the coefficients on both sides of Equation (27), we obtain the following theorem.

Theorem 4. For $n \geq 0$, we have

$$
B_{n+1}(x \mid \lambda)=x \sum_{k=0}^{n} \sum_{l=0}^{k}\left(\begin{array}{l}
n \\
k
\end{array}\right)(x-\lambda)_{l, \lambda} S_{2}(k, l)=x \sum_{k=0}^{n}\left(\begin{array}{l}
n \\
k
\end{array}\right) B_{k}(x-\lambda \mid \lambda) .
$$

In particular,

$$
B_{n+1}(1 \mid \lambda)=\sum_{k=0}^{n}\left(\begin{array}{l}
n \\
k
\end{array}\right) B_{k}(1-\lambda \mid \lambda)
$$

Note that

$$
B_{n+1}(x)=\lim _{\lambda \rightarrow 0} B_{n+1}(x \mid \lambda)=x \sum_{k=0}^{n}\left(\begin{array}{l}
n \\
k
\end{array}\right) B_{k}(x) .
$$

For $n \in \mathbb{N}$, by Theorem 3, we get

$$
\begin{aligned}
B_{n}(x \mid \lambda) & =\sum_{l=1}^{\infty} \sum_{m=1}^{l}\left(\begin{array}{c}
l \\
m
\end{array}\right)(-1)^{l-m} m^{n} \frac{1}{l !}(x)_{l, \lambda} \\
& =\sum_{l=1}^{\infty} \sum_{m=0}^{l-1}\left(\begin{array}{c}
l \\
m+1
\end{array}\right)(-1)^{l-1-m}(m+1)^{n} \frac{1}{l !}(x)_{l, \lambda} \\
& =\sum_{l=1}^{\infty} \sum_{m=0}^{l-1} \frac{1}{(m+1) !(l-m-1) !}(-1)^{l-1-m}(m+1)^{n}(x)_{l, \lambda} \\
& =\sum_{l=0}^{\infty} \sum_{m=0}^{l} \frac{1}{m !(l-m) !}(-1)^{l-m}(m+1)^{n-1}(x)_{l+1, \lambda} \\
& =\sum_{l=0}^{\infty}\left(\frac{1}{l !} \sum_{m=0}^{l}\left(\begin{array}{c}
l \\
m
\end{array}\right)(-1)^{l-m}(m+1)^{n-1}\right)(x)_{l+1, \lambda} .
\end{aligned}
$$


From Equation (5), we note that

$$
\begin{aligned}
\sum_{n=l}^{\infty} S_{2}(n, l \mid 1) \frac{t^{n}}{n !} & =\frac{1}{l !}\left(e^{t}-1\right)^{l} e^{t}=\frac{1}{l !} \sum_{m=0}^{l}\left(\begin{array}{c}
l \\
m
\end{array}\right)(-1)^{l-m} e^{m t} \cdot e^{t} \\
& =\frac{1}{l !} \sum_{m=0}^{l}\left(\begin{array}{c}
l \\
m
\end{array}\right)(-1)^{l-m} e^{(m+1) t} \\
& =\sum_{n=0}^{\infty}\left(\frac{1}{l !} \sum_{m=0}^{l}\left(\begin{array}{c}
l \\
m
\end{array}\right)(-1)^{l-m}(m+1)^{n}\right) \frac{t^{n}}{n !} .
\end{aligned}
$$

By comparing the coefficients on both sides of Equation (29), we get

$$
\frac{1}{l !} \sum_{m=0}^{l}\left(\begin{array}{c}
l \\
m
\end{array}\right)(-1)^{l-m}(m+1)^{n}=\left\{\begin{array}{cl}
S_{2}(n, l \mid 1), & \text { if } n \geq l, \\
0, & \text { if } n<l .
\end{array}\right.
$$

From Equations (28) and (30), we have

$$
B_{n}(x \mid \lambda)=\sum_{l=0}^{n-1} S_{2}(n-1, l \mid 1)(x)_{l+1, \lambda}=\sum_{l=1}^{n} S_{2}(n-1, l-1 \mid 1)(x)_{l, \lambda} .
$$

Therefore, by Equation (31), we obtain the following theorem.

Theorem 5. For $n \in \mathbb{N}$, we have

$$
B_{n}(x \mid \lambda)=\sum_{l=1}^{n} S_{2}(n-1, l-1 \mid 1)(x)_{l, \lambda}
$$

In particular,

$$
B_{n}(1 \mid \lambda)=\sum_{l=1}^{n} S_{2}(n-1, l-1 \mid 1)(1)_{l, \lambda}
$$

Note that

$$
B_{n}(x)=\lim _{\lambda \rightarrow 0} B_{n}(x \mid \lambda)=x \sum_{l=0}^{n-1} S_{2}(n-1, l \mid 1) x^{l}=\sum_{l=1}^{n} S_{2}(n-1, l-1 \mid 1) x^{l} .
$$

By replacing $t$ by $\log (1+t)$ in Equation (22), we get

$$
\begin{aligned}
e_{\lambda}^{x}(t) & =\sum_{m=0}^{\infty} B_{m}(x \mid \lambda) \frac{1}{m !}(\log (1+t))^{m}=\sum_{m=0}^{\infty} B_{m}(x \mid \lambda) \sum_{n=m}^{\infty} S_{1}(n, m) \frac{t^{n}}{n !} \\
& =\sum_{n=0}^{\infty}\left(\sum_{m=0}^{n} S_{1}(n, m) B_{m}(x \mid \lambda)\right) \frac{t^{n}}{n !} .
\end{aligned}
$$

On the other hand,

$$
e_{\lambda}^{x}(t)=\sum_{n=0}^{\infty}(x)_{n, \lambda} \frac{t^{n}}{n !} .
$$

Therefore, by Equations (32) and (33), we obtain the following theorem.

Theorem 6. For $n \geq 0$, we have

$$
(x)_{n, \lambda}=\sum_{m=0}^{n} S_{1}(n, m) B_{m}(x \mid \lambda) .
$$

Note that the identity in Theorem 6 is an inverse to that in Theorem 1. 
We observe that

$$
\begin{aligned}
\frac{d}{d x} e_{\lambda}^{x}\left(e^{t}-1\right) & =\frac{d}{d x} e^{\frac{x}{\lambda} \log \left(1+\lambda\left(e^{t}-1\right)\right)} \\
& =\frac{1}{\lambda} \log \left(1+\lambda\left(e^{t}-1\right)\right)\left(1+\lambda\left(e^{t}-1\right)\right)^{\frac{x}{\lambda}} \\
& =\sum_{l=1}^{\infty} \frac{(-\lambda)^{l-1}}{l}\left(e^{t}-1\right)^{l} \sum_{k=0}^{\infty}(x)_{k, \lambda} \frac{1}{k !}\left(e^{t}-1\right)^{k} \\
& =\sum_{m=1}^{\infty} \sum_{l=1}^{m} \frac{(-\lambda)^{l-1}}{l}(x)_{m-l, \lambda} \frac{m !}{(m-l) !} \frac{1}{m !}\left(e^{t}-1\right)^{m} \\
& =\sum_{m=1}^{\infty} \sum_{l=1}^{m} \frac{(-\lambda)^{l-1}}{l}(x)_{m-l, \lambda} \frac{m !}{(m-l) !} \sum_{n=m}^{\infty} S_{2}(n, m) \frac{t^{n}}{n !} \\
& =\sum_{n=1}^{\infty}\left(\sum_{m=1}^{n} \sum_{l=1}^{m}(-\lambda)^{l-1}(x)_{m-l, \lambda}(l-1) !\left(\begin{array}{c}
m \\
l
\end{array}\right) S_{2}(n, m)\right) \frac{t^{n}}{n !} .
\end{aligned}
$$

On the other hand, from Equation (22), we note that

$$
\frac{d}{d x} e_{\lambda}^{x}\left(e^{t}-1\right)=\sum_{n=0}^{\infty} \frac{d}{d x} B_{n}(x \mid \lambda) \frac{t^{n}}{n !} .
$$

By Equations (34) and (35), we get

$$
\frac{d}{d x} B_{n}(x \mid \lambda)=\sum_{m=1}^{n} \sum_{l=1}^{m}(-\lambda)^{l-1}(x)_{m-l, \lambda}(l-1) !\left(\begin{array}{c}
m \\
l
\end{array}\right) S_{2}(n, m) .
$$

Note that

$$
\lim _{\lambda \rightarrow 0} \frac{d}{d x} B_{n}(x \mid \lambda)=m \sum_{m=1}^{n} x^{m-1} S_{2}(n, m) .
$$

Lastly, we would like to compare the new type degenerate Bell polynomials $B_{n}(x \mid \lambda)$ with the partially degenerate Bell polynomials $\beta_{n, \lambda}(x)$. From Theorem 2, we have

$$
B_{n}(x \mid \lambda)=\sum_{m=0}^{n} \sum_{l=0}^{m} x^{l} \lambda^{m-l} S_{1}(m, l) S_{2}(n, m) .
$$

On the other hand, from Equation (9) and Proposition 3, we obtain

$$
\beta_{n, \lambda}(x)=\sum_{m=0}^{n} \sum_{l=0}^{m} x^{l} \lambda^{n-m} S_{1}(n, m) S_{2}(m, l) .
$$

By simple computations using Equations (37) and (38), we can show that

$$
\begin{aligned}
B_{0}(x \mid \lambda)= & \beta_{0, \lambda}(x)=1, \quad B_{1}(x \mid \lambda)=\beta_{1, \lambda}(x)=x, \\
B_{2}(x \mid \lambda)= & \beta_{2, \lambda}(x)=-x \lambda+\left(x^{2}+x\right), \\
B_{3}(x \mid \lambda)= & \beta_{3, \lambda}(x)=2 x \lambda^{2}+\left(-3 x^{2}-3 x\right) \lambda+\left(x^{3}+3 x^{2}+x\right), \\
B_{4}(x \mid \lambda)= & -6 x \lambda^{3}+\left(11 x^{2}+12 x\right) \lambda^{2}+\left(-6 x^{3}-18 x^{2}-7 x\right) \lambda+\left(x^{4}+6 x^{3}+7 x^{2}+x\right), \\
\beta_{4, \lambda}(x)= & -6 x \lambda^{3}+\left(11 x^{2}+11 x\right) \lambda^{2}+\left(-6 x^{3}-18 x^{2}-6 x\right) \lambda+\left(x^{4}+6 x^{3}+7 x^{2}+x\right), \\
B_{5}(x \mid \lambda)= & 24 x \lambda^{4}+\left(-50 x^{2}-60 x\right) \lambda^{3}+\left(35 x^{3}+110 x^{2}+50 x\right) \lambda^{2} \\
& +\left(-10 x^{4}-60 x^{3}-75 x^{2}-15 x\right) \lambda+\left(x^{5}+10 x^{4}+25 x^{3}+15 x^{2}+x\right), \\
\beta_{5, \lambda}(x)= & 24 x \lambda^{4}+\left(-50 x^{2}-50 x\right) \lambda^{3}+\left(35 x^{3}+105 x^{2}+35 x\right) \lambda^{2} \\
& +\left(-10 x^{4}-60 x^{3}-70 x^{2}-10 x\right) \lambda+\left(x^{5}+10 x^{4}+25 x^{3}+15 x^{2}+x\right) .
\end{aligned}
$$




\section{Conclusions}

In the present paper, the new type degenerate Bell polynomials $B_{n}(x \mid \lambda)$ (see Equation (22)) are introduced, which are different from the previously defined partially degenerate Bell polynomials $\beta_{n, \lambda}(x)$ (see Equation (3)) and a degenerate version of the ordinary Bell polynomials $B_{n}(x)$ (see Equation (1)).

In order to compare some properties of the new type degenerate Bell polynomials with those of the partially degenerate Bell polynomials, we first reviewed several expressions for the latter which include an expression involving the degenerate Stirling numbers of the second kind, Dobinski type formula, and an expression connected with the degenerate central factorial numbers of the second kind. In addition, we gave identities relating $\beta_{n, \lambda}(x)$ to $B_{n}(x)$.

As to the new type degenerate Bell polynomials, we obtained results which are parallel to those of the partially degenerate Bell polynomials. Namely, we obtained an expression involving the Stirling numbers of the second kind and the generalized falling factorial sequences, Dobinski type formulas, an expression connected with the Stirling numbers of the first and second kinds, and an expression involving the Stirling polynomials of the second kind. Further, we derived a recursive formula for them.

As we mentioned in the Introduction, studying degenerate versions of some special polynomials can be traced back at least as early as the paper [1] by Carlitz and regained interests of many mathematicians in recent years. It should be noted that the idea of a degenerate version has also been explored in dynamics [26]. We also noticed that this idea of studying degenerate versions of special polynomials is not just limited to polynomials but can be extended to transcendental functions like gamma functions [10,11].

This line of research has been very fruitful, as it was demonstrated, for example, by the papers [2-9]. We would like to continue this line of research as one of our research projects. In more detail, we will study degenerate versions of many special polynomials and numbers and those of some transcendental functions, and investigate their applications to mathematics, physics and engineering as well as their number theoretic and combinatorial properties.

Author Contributions: T.K. and D.S.K. conceived the framework and structured the whole paper; T.K. and D.S.K. wrote the paper; J.K. and H.L. checked the results of the paper; D.S.K. and T.K. completed the revision of the article.

Funding: This work was supported by the National Research Foundation of Korea (NRF) grant funded by the Korea government (MEST) (No. 2017R1E1A1A03070882).

Acknowledgments: The authors would like to thank the referees for their helpful comments and suggestions.

Conflicts of Interest: The authors declare no conflict of interest.

\section{References}

1. Carlitz, L. Degenerate Stirling, Bernoulli and Eulerian numbers. Util. Math. 1979, 15, 51-88.

2. Dolgy, D.V.; Jang, G.-W.; Kim, T. A note on degenerate central factorial polynomials of the second kind. Adv. Stud. Contemp. Math. (Kyungshang) 2019, 29, 7-13.

3. Jeong, J.; Rim, S.-H.; Kim, B.M. On finite-times degenrate Cauthy numbers and polynomials. Adv. Diff. Equ. 2015, 321, 12 .

4. Kim, D.S.; Kim, T.; Jang, G.-W. A note on degenerate Stirling numbers of the first kind. Proc. Jangjeon Math. Soc. 2018, 21, 393-404.

5. Kim, T.; Kim, D.S. Degenerate central factorial numbers of the second kind. Revista de la Real Academia de Ciencias Exactas Físicas y Naturales Serie A Matemáticas 2019, 113, 3359-3367. [CrossRef]

6. Kim, T.; Kim, D.S.; Jang, L.-C.; Kwon, H.-I. Extended degenerate Stirling numbers of the second kind and extended degenerate Bell polynomials. Util. Math. 2018, 106, 11-21.

7. Kim, T. A note on degenerate Stirling numbers of the second kind. Proc. Jangjeon Math. Soc. 2017, 20, 319-331.

8. Lee, J.G.; Kim, W.J.; Jang, L.-C.; Kim, B.M. A note on modified degenerate $q$-Daehee polynomials and numbers. J. Inequal. Appl. 2019, 2019, 24. [CrossRef] 
9. Pyo, S.S. Degenerate Cauthy numbers and polynomials of the fourth kind. Adv. Stud. Contemp. Math. (Kyungshang) 2018, 28, 127-138.

10. Kim, T.; Jang, G.-W. A note on degenerate gamma function and degenerate Stirling number of the second kind. Adv. Stud. Contemp. Math. (Kyungshang) 2018, 28, 207-214.

11. Kim, T.; Kim, D.S. Degenerate Laplace transform and degenerate gamma function. Russ. J. Math. Phys. 2017, 24, 241-248. [CrossRef]

12. Bell, E.T. Exponential polynomials. Ann. Math. 1934, 35, 258-277. [CrossRef]

13. Comtet, L. Advanced Combinatorics: The Art of Finite and Infinite Expansions; Translated from the Frech by J.W. Nienhuys; Reidel: Dordrecht, The Netherlands; Boston, MA, USA, 1974.

14. Kim, T.; Kim, D.S.; Dolgy, D.V. On partially degenerate Bell numbers and polynomials. Proc. Jangjeon Math. Soc. 2017, 20, 337-345.

15. Kim, T.; Kim, D.S.; Kwon, H.-I.; Rim, S.-H. Some identities for umbral calculus associated with partially degenerate Bell numbers and polynomials. J. Nonlinear Sci. Appl. 2017, 10, 2966-2975. [CrossRef]

16. Brillhart, J. Mathematical Notes: Note on the Single variable Bell polynomials. Am. Math. Mon. 1967, 74, 695-696. [CrossRef]

17. Brarman, F. On Touchard polynomials. Can. J. 1957, 9, 191-193.

18. Carlitz, L. Some arithmetic properties of the Bell polynomials. Bull. Am. Math. Soc. 1965, 71, 143-144. [CrossRef]

19. Carlitz, L. Arithmetic properties of the Bell polynomials. J. Math. Anal. Appl. 1966, 15, 33-52. [CrossRef]

20. Kim, D.S.; Kim, T. Some identities of Bell polynomials. Sci. China Math. 2015, 58, 2095-2104. [CrossRef]

21. Roman, S. The Umbral Calculus, Pure and Applied Mathematics; Academic Press, Inc. [Harcourt Brace Jovanovich, Publishers]: New York, NY, USA, 1984.

22. He, Y.; Pan, J. Some recursion formulae for the number of derangements and Bell numbers. J. Math. Res. Appl. 2016, 36, 15-22.

23. Shang, Y. Localized recovery of complex networks against failure. Sci. Rep. 2016, 6, 30521. [CrossRef] [PubMed]

24. Shang, Y. False positive and false negative effects on network attacks. J. Stat. Phys. 2018, 170, $141-164$. [CrossRef]

25. Zhang, W. Some identities involving the Euler and the central factorial numbers. Fibonacci Quart. 1998, 36, 154-157.

26. Shang, Y. Deffuant model with general opinion distributions: First impression and critical confidence bound. Complexity 2013, 19, 38-49. [CrossRef] 